\title{
Author Correction: Genetic circuit design automation for the gut resident species Bacteroides thetaiotaomicron
}

Mao Taketani, Jianbo Zhang (D), Shuyi Zhang, Alexander J. Triassi@, Yu-Ja Huang, Linda G. Griffith and

Christopher A. Voigt (10)

Correction to: Nature Biotechnology https://doi.org/10.1038/s41587-020-0468-5, published online 30 March 2020.

In the version of this article initially published, the second paragraph of main text stated that the concentration of bile acids in the human large intestine is $\sim 500 \mathrm{mM}$. The correct value is $\sim 500 \mu \mathrm{M}$. The error has been corrected in the HTML and PDF versions of the article.

Published online: 7 May 2020

https://doi.org/10.1038/s41587-020-0545-9

() The Author(s), under exclusive licence to Springer Nature America, Inc. 2020

\section{Author Correction: Plants with genetically encoded autoluminescence}

Tatiana Mitiouchkina, Alexander S. Mishin (D), Louisa Gonzalez Somermeyer (D), Nadezhda M. Markina, Tatiana V. Chepurnyh, Elena B. Guglya, Tatiana A. Karataeva, Kseniia A. Palkina, Ekaterina S. Shakhova, Liliia I. Fakhranurova, Sofia V. Chekova, Aleksandra S. Tsarkova, Yaroslav V. Golubev, Vadim V. Negrebetsky, Sergey A. Dolgushin, Pavel V. Shalaev, Dmitry Shlykov, Olesya A. Melnik, Victoria O. Shipunova, Sergey M. Deyev, Andrey I. Bubyrev, Alexander S. Pushin, Vladimir V. Choob, Sergey V. Dolgov, Fyodor A. Kondrashov, Ilia V. Yampolsky and Karen S. Sarkisyan (D)

Correction to: Nature Biotechnology https://doi.org/10.1038/s41587-020-0500-9, published online 27 April 2020.

In the version of this article initially published online, a sentence was missing from the Acknowledgements section: The authors would like to acknowledge the work of Genomics Core Facility of the Skolkovo Institute of Science and Technology, which performed the sequencing and bioinformatic analysis. The error has been corrected in the HTML and PDF versions of the article.

Published online: 4 June 2020

https://doi.org/10.1038/s41587-020-0578-0

(c) The Author(s), under exclusive licence to Springer Nature America, Inc. 2020

\section{Publisher Correction: The hothouse for protein design}

Caroline Seydel

Correction to: Nature Biotechnology https://doi.org/10.1038/s41587-020-0586-0, published online 23 June 2020.

In the version of this article initially published, a merger between Neoleukin Therapeutics and Aquinox Pharmaceuticals was described as an acquisition of the former by the latter. Specifically, the last sentence of section "Eliminating side effects" originally read "That August, the company was purchased by Vancouver-based Aquinox Pharmaceuticals, in a deal worth around $\$ 40$ million." It has been changed to "That August, the company merged with Vancouver-based Aquinox Pharmaceuticals to become a publicly traded entity, the first IPD spinout to go public, under the name Neoleukin Therapeutics." The error has been corrected in the HTML and PDF versions of the article.

Published online: 22 July 2020

https://doi.org/10.1038/s41587-020-0641-X

(c) The Author(s), under exclusive licence to Springer Nature America, Inc. 2020 